\title{
Patient-related factors associated with poor glycaemic control among patients with type 2 diabetes mellitus
}

Pablo Romakin,

Masoud Mohammadnezhad

\section{Background and objective Poorly controlled type 2 diabetes mellitus (T2DM) is the number one cause of premature deaths, hospital admissions and disability in Fiji. The purpose of this qualitative study was to determine healthcare providers' perceptions of factors associated with glycaemic control.}

\section{Methods}

Three focus group discussions were conducted with 19 healthcare providers selected through purposive variation sampling in three selected diabetes clinics in Suva, Fiji, in August 2017 and analysed using thematic analysis.

\section{Results}

Five themes on factors associated with poor glycaemic control emerged: patients' adherence to treatment and management plans, attitudes, knowledge about diabetes, culture/beliefs and support. Other factors that play a pivotal part in achieving good glycaemic control are healthcare providers' behaviour and addressing patient's caregiver issues.

\section{Discussion}

Knowing how to empower patients with T2DM to manage the different patientrelated factors associated with poor glycaemic control at first encounter will assist healthcare providers to deliver high-quality patient-centred diabetic care services and achieve better health outcomes in patients.
DIABETES MELLITUS is one of the most common health problems, reaching epidemic proportions globally. One in 11 people is affected by the condition, which had a prevalence rate of $8.8 \%$ in $2017 .{ }^{1}$ In Fiji, diabetes mellitus has reached epidemic proportions, with a prevalence rate of $15.6 \%$ in 2011 , which is projected to rise to $19.3 \%$ in $2020 .^{2,3}$ This high prevalence rate is caused by high rates of obesity arising from changes in lifestyle factors due to urbanisation..$^{4,5}$ Diabetes mellitus is the number one cause of disease-specific mortality, premature deaths and most disability from 2005-16. ${ }^{6}$ The Fiji Non-Communicable Disease (NCD) Steps Survey 2002 found that $32.2 \%$ of people aged $25-64$ years who were previously diagnosed with diabetes and are taking medication for the condition have poor blood sugar control. $^{7}$

Type 2 diabetes mellitus (T2DM), which is the focus of this study, is the most common type of diabetes mellitus and accounts for $90-95 \%$ of all diabetes cases. ${ }^{8,9}$ It is defined as a heterogeneous metabolic disorder characterised by hyperglycaemia secondary to impaired insulin secretion, defective insulin action or combination of both. ${ }^{10,11}$ Poor glycaemic control among patients with T2DM results in a great financial burden both on the patient and the Fiji health system, where the costs of managing patients with T2DM are more than double, compared with patients without the condition, because of the complications associated with T2DM. ${ }^{12,13}$

Healthcare providers' perceptions of factors associated with poor glycaemic control in patients with T2DM have been shown to have a significant impact on patient management, ${ }^{14,15}$ with studies showing that patient characteristics ${ }^{16,17}$ and behaviours are major barriers to achieving good glycaemic control. ${ }^{18,19}$ There has been remarkable progress in terms of development of numerous effective diabetes management tools and interventions; however, significant gaps still exist between knowledge gained through research and the day-to-day clinical practice to support patients with T2DM to achieve good glycaemic control. ${ }^{20}$ Therefore, the purpose of this qualitative study was to determine healthcare providers' perceptions of patient-related factors associated with glycaemic control among patients with T2DM attending clinics at three selected health centres in Suva, Fiji, in 2017.

The findings of this study will be beneficial to the Fiji Ministry of Health and Medical Services (MOHMS) because they will increase the understanding of healthcare providers' perceptions of the influence of patient-related factors on glycaemic control among patients with T2DM. Addressing these issues from the perspectives of the healthcare providers is important, as the healthcare providers make decisions that have a large impact on resource use and patient management outcomes. 


\section{Methods}

This was a qualitative study with an explorative design based on grounded theory that was informed by a social constructionist approach, where a general abstract theory of actions and interactions was constructed out of the views and perceptions of the participants, ${ }^{21}$ who were trying to explain their experiences of dealing with glycaemic control of patients with T2DM attending their clinics. The researcher used this approach to better understand the healthcare providers' perceptions and actions about glycaemic control in patients with T2DM that are not readily available in clinical settings.

The research questions (Table 1) were based on the researcher's extensive experience as a medical practitioner who is frustrated by the fact that, despite what is known about glycaemic control and the availability of a wide range of therapeutic options, there is still a high prevalence rate of poor glycaemic control among patients with T2DM in Fiji, who have preventable complications.

Although the researcher had prior knowledge of and experience with T2DM, he was unaware of what the participants would bring to the study. As the participants' manager, the researcher distanced himself from the participants by hiring a research assistant who facilitated the focus group discussion (FGD).

The settings of the study were three selected health centres with diabetes clinics in Suva, Fiji:

- Lami health centre, a facility that predominantly looks after a rural and semi-urban population
- Valelevu health centre, a facility that looks after an urban population and those who live in informal settlements

- Suva diabetes centre, a health facility with a multidisciplinary team managing patients with T2DM.

These facilities were chosen to allow collection of information from participants with varied backgrounds and experiences, who are caring for patients with T2DM of varied sociodemographic characteristics.

This study involved 19 healthcare providers selected through purposive variation sampling from three randomly selected health centres in Suva, Fiji. It was conducted in August 2017. The participants were selected using the following inclusion criteria:

- healthcare provider currently working in the diabetes clinic

- medical officer in-charge

- nurse team leader of the selected health centres.

Three FGDs facilitated by a research assistant were conducted in each selected health centre. An information sheet that included details of the study and its benefits, risks, disadvantages and confidentiality was given and explained to the participants before they signed a consent form. A demographic characteristic form was completed by the participants to collect sociodemographic information. A digital audio recorder was used to record the FGDs. The number of participants for the FGDs ranged from five to seven participants, with a median of six participants. Each FGD session lasted for 60 minutes. To maintain confidentiality, the participants were addressed according

\section{Table 1. Research questions}

\begin{tabular}{ll}
\hline No & Research question \\
\hline 1 & $\begin{array}{l}\text { What are the perspectives of healthcare providers about glycaemic control } \\
\text { among patients with type } 2 \text { diabetes mellitus (T2DM)? }\end{array}$ \\
\hline 2 & $\begin{array}{l}\text { How can a healthcare provider contribute to glycaemic control among patients } \\
\text { with T2DM? }\end{array}$ \\
\hline 3 & $\begin{array}{l}\text { What are the patient-related factors that contribute to poor glycaemic control } \\
\text { among patients with T2DM? }\end{array}$ \\
\hline
\end{tabular}

to their assigned codes. The participants were asked six semi-structured open-ended questions with prompts. These questions were engagement, exploration and exit questions formulated from the research questions of this study after a brainstorming session (Table 2). The questions were pre-tested with healthcare providers of a diabetes clinic that was not chosen for this study. All necessary approvals were given by the College Health Research and Ethics Committee (CHREC) of Fiji National University and the Fiji National Health Research and Ethics Review Committee (FNHRERC) before commencing the study (Approval number: 2017.106.C.D).

The qualitative data collected from the FGDs (conducted during the period 1-30 August 2017) were transcribed by the researcher from the digital audio recorder using handwritten notes, copied to the electronic Microsoft Word 2010 format and sent to the research assistant for verification. The verified transcripts were entered into a Microsoft Excel spreadsheet. Key words and phrases that suggested significant meanings were identified and grouped. All data were then coded. Data coding and categorisation continued until theoretical saturation was reached. Data were thematically analysed using Attride-Stirling's thematic network analysis framework of basic, organising and global themes. ${ }^{22}$ The resulting themes formed the structure of the results portion. Quotes from participants were used to illustrate responses related to the relevant themes.

To ensure trustworthiness in reporting the findings of this study, credibility was ensured by interviewing the participants about their perceptions and experiences in their own clinics. After encoding, the interview transcripts were returned to the FGD facilitator to ensure accuracy of the codes and the relevant interpretations. Dependability was established through detailed data analysis and direct professional experience of the individual participants. A description of the sociodemographic characteristics of the participants and the data collection and analysis processes are presented for the reader to determine whether the findings would be transferrable to other settings. 


\section{Results}

\section{Sociodemographic characteristics of participants}

The ages of the participants ranged from 28 to 58 years (mean $=39$ years, standard deviation $= \pm 9.2$ years) with the majority in the age group 30-39 years. The majority of the participants were nurses (52.6\%). The sociodemographic characteristics of the participants are presented in Table 3.

\section{Themes}

Five themes on patient-related factors associated with poor glycaemic control were identified from the FGDs: patients' adherence to treatment and management plans, patients' attitudes towards self-care, patients' knowledge about diabetes, patients' culture/beliefs and patient support.

\section{Theme 1. Patients' adherence to} treatment and management plans Poor adherence to treatment and prescribed diabetic diet and lack of physical activity were mentioned by 13 participants $(n=13 / 19)$ as factors associated with poor glycaemic control.

The majority of participants mentioned that one major factor that influences adherence to treatment among their patients with T2DM is the healthcare provider's attitude. They believed that the healthcare provider's behaviour - for example, poor patient-healthcare provider relationship (lack of trust and poor rapport), rushed patient consultation time and lack of motivational skills - contributes to patients' lack of motivation.

\section{One major factor that influences patients'} adherence to treatment is our [healthcare providers'] attitude. We need to spend more time with them to identify all the issues that will affect their treatment. Ah, we should be showing a genuine attitude while treating them, the reassurance that we give them. We need to gain their trust. (Participant $10 \mathrm{~b}$, female, diabetes clinic nurse)

Other factors causing poor adherence to treatment and diabetes management plans were:

- defaulting clinic appointments, where the participants believed this was due to financial issues, disability or limitations in mobility, acute illness and lack of flexibility in clinic appointments

- medication stock-outs

- the patient's lack of understanding of the diabetes disease process

- polypharmacy, including medication side effects.

Theme 2. Patients' attitudes towards self-care Twelve healthcare providers $(n=12 / 19)$ identified patients' attitudes towards their self-care as one of the contributing factors to having poor glycaemic control. Patients' attitudes include what the participants

Table 2. Focus group discussion semi-structured questionnaire

\begin{tabular}{ll}
\hline Type & Questions \\
\hline Engagement question & $\begin{array}{l}\text { As a healthcare provider in a diabetes clinic, what does } \\
\text { glycaemic or blood sugar control in patients with type } 2 \text { diabetes } \\
\text { mellitus (T2DM) mean to you? } \\
\text { Probe: Please explain further and provide examples. }\end{array}$ \\
\hline
\end{tabular}

Exploration questions

What do you think are the patient-related factors that influence glycaemic control among your patients with T2DM?

Probe: Please elaborate more on these factors and provide examples.

How can you (as a healthcare provider) contribute to glycaemic control among your patients with T2DM?

Probe: Please tell me more about it based on your experience?

termed as a lack of 'motivation' to look after their own health, with too much dependence on the healthcare providers.

Because it all starts with them, whether they are willing to take our advice to take the medications that we give them. So, I think, it's [patients' motivation that is] the most important. (Participant 8b, female, medical officer)

Another type of attitude identified was patients' refusal of treatment (eg patient does not want to inject herself with insulin) as well as competing priorities, where health is not a priority for the majority of patients coming to the clinic.

Theme 3. Patients' knowledge about diabetes Fourteen participants $(n=14 / 19)$ reported that patients with T2DM lack or have very limited knowledge about diabetes, its complications and treatment goals. They believe that poor educational backgrounds and literacy levels are the main barriers in patients acquiring knowledge about diabetes, glycaemic control and preventing complications. Denial of the condition, stress and a wide range of mental health issues that are associated with chronic conditions such as diabetes were identified as limiting factors in receiving knowledge.

The way in which patients receive diabetes knowledge also depends on the healthcare workers. Language barriers and a lack of direction from healthcare workers were also identified as barriers to receiving knowledge.

The way we [healthcare providers] talk and explain things matters a lot. So, in this sense, for the patients, language matters. If they don't understand what we are saying, they'll get it wrong. (Participant 6b, female, medical officer in-charge)

Theme 4. Patients' culture and beliefs The majority of participants $(n=13 / 19)$ observed that culture and traditions influence glycaemic control.

You know after Diwali Celebration [a Hindu festival], patients come with very high blood sugar level after eating too [many] sweets.

(Participant 16c, female, nurse) 
They also identified that some patients still practise herbal and traditional medicines to lose weight and cure any chronic illness such as cancer and diabetes. The participants also found that patients believe in the power of prayer and 'miracle water' to cure whatever illness they have.

I often see patients with [a] very bad, foul-smelling and gangrenous foot where patients use coconut oil and pounded leaves ... as dressing to their wounds. (Participant $7 \mathrm{~b}$, female, nurse)

\section{Theme 5. Patient support}

In this study, patient support was a recurrent theme during discussions, as identified by 14 of the participants ( $n=14 / 19)$. Most participants believed that their patients live in an extended family and close-knit community where support is always there. However, patient support systems are also affected when their quality of life (both personal and work situations) is affected by social problems, stress, frustrations and fatigue due to the demanding nature of caring for their sick relatives. Family members and treatment support groups are not usually involved during patient consultations as healthcare providers see them as a hindrance to patient care.

You know, most of the diabetes patients we have are very demanding and difficult to take care [of]. Sometimes, their family members are frustrated and do not know what to do with them. (Participant 2a, male, charge nurse)

The majority of participants acknowledged that patient-related factors contributing to glycaemic control among patients with T2DM are beyond their control, but they believe that healthcare providers can influence these factors by providing patients with good knowledge on diabetes care and management, a

\section{Table 3. Participant sociodemographic characteristics}

\section{Participant characteristics $(n=19)$}

n (\%)

\section{Professional cadre}

\begin{tabular}{lr}
\hline Medical officer & $6(31.6)$ \\
\hline Nurse practitioner & $1(5.3)$ \\
\hline Nurse & $10(52.6)$ \\
\hline Dietitian & $2(10.5)$ \\
\hline Age (years) & $3(15.8)$ \\
\hline$<30$ & $8(42.1)$ \\
\hline $30-39$ & $6(31.6)$ \\
\hline $40-49$ & $2(10.5)$
\end{tabular}

\section{Sex}

\section{Female} $16(84.2)$

\section{Male}

$3(15.8)$

\section{Length of time working at diabetes clinic (years)}

\begin{tabular}{rr}
\hline$<1$ & $5(26.3)$ \\
\hline $1-5$ & $4(21.1)$ \\
\hline$>5$ & $10(52.6)$ \\
\hline
\end{tabular}

caring attitude and competent skills to educate and motivate patients towards treatment compliance, healthy lifestyles and self-care as well as having a supportive health system.

\section{Discussion}

This qualitative study has explored key perceptions and experiences of healthcare providers on the different patient-related factors that influence glycaemic control among patients with T2DM attending their clinics. It has provided valuable information to support healthcare providers to deliver high-quality diabetic services for patients with T2DM to achieve good glycaemic control.

In this study, healthcare providers perceived the following patient-related factors as contributing to poor glycaemic control among patients with T2DM: poor adherence to treatment and management plans; patients' attitudes (which were described as a lack of motivation to look after their own health and total dependence on the healthcare workers); patients' lack of knowledge about diabetes, its complications and treatment goals; patients' culture and beliefs and preference to use traditional medicines; and lack of patient support. This aligns with other qualitative studies investigating the perceptions of primary care providers, which have found that patients' poor motivation, resistance to lifestyle-modifying behaviours, poor treatment adherence and awareness, and cultural beliefs and attitudes are the main barriers to optimal glycaemic control. ${ }^{23-25}$ These findings are not only common in Fiji and other low-income to middle-income countries, but also in wealthier countries such as Australia. ${ }^{26}$

Studies show that patient characteristics play a significations part in glycaemic control. Le Blanc et al described in their study of 149 primary care providers, which included physicians, nurse practitioners and pharmacy assistants in the US, that patient characteristics, but not provider characteristics, predicted adherence to treatment and glycaemic control. They suggested that improving diabetes care systems and services provides better 
health outcomes among patient with T2DM than focusing on individual healthcare providers. ${ }^{16}$ Houle et al, after conducting a prospective observational study on 295 patients with T2DM in diabetes clinics in California, found that improvements in diet, medication-taking and self-monitoring of glucose lead to improvements in glycaemic control. ${ }^{17}$ However, in the present study, the majority of participants believed that healthcare providers greatly influence and have a pivotal role in patients' adherence to treatment and management plans. Good patient-healthcare provider communication and a relationship that is built on trust, mutual respect and being sensitive to patients' personal and cultural beliefs ensures adherence to treatment. They believe that through these attributes, healthcare providers will be able to help patients discover and develop their inherent capacity to look after their own health. This is supported by the results of the study conducted by Wens et al on 40 general practitioners (GPs) in Belgium, which found that GPs need good communication skills to cope with T2DM expectations and achieve better treatment outcomes. ${ }^{27}$ As the majority of their patients are elderly and live with extended family, the participants in this cohort believed that treatment support, whether it comes in the form of family, friends, community or a peer support group, is essential to ensure patients' adherence to treatment.

Glycaemic control among patients with $\mathrm{T} 2 \mathrm{DM}$ is dependent on how the patient behaves. Most studies have shown that self-efficacy, motivation and attitude play a key part in patients' self-care. ${ }^{18,19,28,29}$ In this study, participants mentioned that the majority of their patients with T2DM are women who live in a paternalistic society where men are still the powerful figure in the family. These women are dependent on their family for food, exercise, when to go to the clinic, whether or not to take their medications and for support. They are not expected to make personal decisions. It is very important for healthcare providers to understand this, as it hinders patient engagement and reduces motivation toward self-care.
Studies have shown that when patients with T2DM have a basic understanding of the treatment goals and what can be done to achieve these goals, they are able to adhere to self-care management and achieve their glycaemic targets. Patients with chronic disease who are actively involved in their healthcare through patient empowerment and effective collaborative relationships with their healthcare providers have better health outcomes. ${ }^{30,31}$ This study found, however, that according to the participants, the majority of their patients with poorly controlled T2DM have minimal understanding of their diabetes treatment goals and targets. The majority of the patients are totally dependent on their healthcare providers to control their blood sugar for them. When something goes wrong, patients blame their healthcare providers for not giving the right medications and/or the right dosage.

According to the World Health Organization, between $60 \%$ and $80 \%$ of the population use traditional medicine in Fiji. ${ }^{32}$ Traditional medicines, especially herbal formulations, are preferred by many patients with $\mathrm{T} 2 \mathrm{DM}$ because of the general perceptions of having fewer side effects and lower cost when compared with antidiabetic medications. ${ }^{33}$ In this study, the majority of the participants mentioned that most of their patients prefer herbal medications over antidiabetic medications or insulin because they are easily accessible, free and do not have side effects. Many patients take herbal medications because somebody told them that these herbal medications were proven to be effective to control blood sugar, prevent diabetes complications and totally cure diabetes, in contrast to healthcare providers saying that diabetes can only be controlled but not totally cured. It is therefore necessary for healthcare providers to ask patients routinely about the use of herbal or alternative medicines to better understand the reasons for their use. This also provides an opportunity for healthcare providers to better communicate with their patients and provide responsible healthcare.

Earlier qualitative studies have found that involvement of family and the community in supporting patient care facilitates good glycaemic control among patients with T2DM. ${ }^{34,35}$ In this study, according to the participants, the majority of the patients with T2DM live with their extended family. There is a very strong sense of family and community support. However, the quality of life of the patients' family members is also affected because of social problems, stress, frustrations and fatigue. This has also affected their caregivers' personal and work situations. Family members and treatment support groups are not usually involved during patient consultations as healthcare providers see them as a hindrance to patient care.

This study found that other factors associated with glycaemic control are beyond patients' and healthcare providers' control, for example, medication and medical consumable stock-outs, financial issues and human resource constraints. Studies conducted in Fiji have shown that shortages of medications, medical consumables and basic medical equipment affect healthcare providers, patients and quality of healthcare delivery. ${ }^{36,37}$

In ignoring patient-related factors, healthcare providers may tend to limit their focus to clinically-oriented issues and may miss many opportunities for effective intervention to achieve good glycaemic control.

\section{Strength}

This study has identified patient-related factors associated with poor glycaemic control among patients with T2DM attending clinics at Suva health centres in Fiji. Understanding these factors will assist healthcare providers with developing strategies that will motivate their patients to actively participate in their care and eventually manage their disease effectively.

\section{Limitations}

The findings of this study must be interpreted in the context of its limitations. This study was conducted among healthcare providers working in urban settings. Selection of participants in rural settings and in remote areas might shed more light on other patient-related 
factors associated with poor glycaemic control. Also, patients' perspectives were not explored to determine the different facilitators, for and barriers to, achieving good glycaemic control.

\section{Conclusion}

Healthcare providers have identified that patient-related factors associated with poor glycaemic control among patients with T2DM include patients' poor adherence to prescribed treatment and management plans; patients' poor attitude (described as lack of motivation to look after their own health, with too much dependence on their healthcare providers); patients' lack of, or limited knowledge of, diabetes, its complications and treatment goals; patients' culture and beliefs on traditional treatment of diabetes; and lack of family, employer and community support.

\section{Implications for general practice}

Healthcare providers in Fiji are often faced with a dilemma that despite their efforts, the majority of their patients with T2DM still have poor glycaemic control and preventable complications. It is therefore necessary for healthcare providers to identify patient-related factors associated with poor glycaemic control among their patients with T2DM for the providers to be able to understand and communicate better with their patients, motivate them to self-care and provide better patientcentred care.

This study also found that patientrelated factors are not the only factors responsible for glycaemic control in T2DM. A variety of factors that are sometimes beyond the healthcare providers' and patients' control also play a part. Further research is needed to identify other key determinants of glycaemic control among patients with T2DM to achieve a more effective diabetes management strategy and glycaemic control, especially in the context of resource limitations.

\section{Authors}

Pablo Romakin MD, MPH, Chief Medical Officer Community Health Suva, Fiji Ministry of Health and Medical Services, Fiji
Masoud Mohammadnezhad BSc, MSc, PhD, Associate Professor in Public Health (Health Promotion), School of Public Health and Primary Care, Fiji National University, Fiji. masoud.m@fnu.ac.fj Competing interests: None.

Funding: None.

Provenance and peer review: Not commissioned, externally peer reviewed.

\section{Acknowledgements}

The authors would like to acknowledge the following for their contribution to this study: staff participants from the three selected health centres for their participation in the focus group discussions; $\mathrm{Dr}$ Donald Wilson for his technical advice and Mrs Sabiha Khan for her advisory input and encouragement.

\section{References}

1. International Diabetes Federation. IDF Diabetes Atlas: Eighth Edition 2017. Brussells: International Diabetes Federation, 2017. p. 40-58. Available at https://diabetesatlas.org/IDF_Diabetes_Atlas_8e_ interactive_EN/ [Accessed 3 May 2019].

2. Morell S, Lin S, Tukana I, et al. Diabetes incidence and projections from prevalence surveys in Fiji. Popul Health Metr 2016;14(45):1-8. doi: 10.1186/ s12963-016-0114-0.

3. Lin S, Tukana I, Linhart C, et al. Diabetes and obesity trends in Fiji over 30years. J Diabetes 2016;8(4):5331-43. doi: 10.1111/1753-0407.12326.

4. Nanditha A, Ma RCW, Ramachandran A, et al. Diabetes in Asia and the Pacific: Implications for the global epidemic. Diabetes Care 2016;39(3):472-85. doi: 10.2337/dc15-1536.

5. Win Tin ST, Lee CMY, Colagiuri R. A profile of diabetes in Pacific Island Countries and Territories. Diabetes Res Clin Pract 2015;107(2):233-46. doi: 10.1016/j.diabres.2014.10.010.

6. Institute for Health Metrics and Evaluation. Fiji. Seattle, WA: University of Washington, 2019. Available at www.healthdata.org/fiji [Accessed 3 May 2019]

7. Ministry of Health. Fiji - Fiji NCD STEPS survey 2002. Suva: Ministry of Health, 2014. Available at www.health.gov.fj/fijindr/index.php/catalog/1 [Accessed 27 June 2019].

8. Professional Practice Committee. Standards of medical care in diabetes - 2018. Diabetes Care 2018;41Suppl 1:S3. doi: 10.2337/dc18-Sppc01.

9. World Health Organization. Global report on diabetes. Geneva: WHO, 2016.

10. Canadian Diabetes Association Clinical Practice Expert Committee, Goldenberg, R, Punthakee, Z. Definition, classification and diagnosis of diabetes, prediabetes and metabolic syndrome. Can J Diabetes 2013;37 Suppl 1:S8-11. doi: 10.1016/j. jcjd.2013.01.011.

11. Karalliedde, J, Gnudi, L. Diabetes mellitus, a complex and heterogeneous disease, and the role of insulin resistance as a determinant of diabetic kidney disease. Nephrol Dial Transplant 2016;31(2):206-13. doi: 10.1093/ndt/gfu405.

12. Zimmet $P$. The burden of type 2 diabetes: Are we doing enough? Diabetes Metab 2003;29(4):6S96S18. doi: 10.1016/S1262-3636(03)72783-9.

13. Baker IDI Heart \& Diabetes Institute. Diabetes: The silent pandemic and its impact on Australia. Baulham Hills, NSW: Novo Nordisk Pharmaceuticals, 2012.

14. Freeman J, Loewe R. Barriers to communication about diabetes mellitus: Patients' and physicians different view of the disease. J Fam Pract 2000:49(6):507-12
15. Ross SA. Breaking down patient and physician barriers to optimize glycaemic control in type 2 diabetes. Am J Med 2013;126(9) Suppl 1:S38-S48. doi: 10.1016/j.amjmed.2013.06.012.

16. Le Blanc E, Rosales G, Kachroo S, Mukherjee J, Funk KL, Nichols GA. Do patient or provider characteristics impact management of diabetes? Am J Manag Care 2015;21(9):597-606.

17. Houle J, Beaulieu MD, Chiasson JL, et al. Glycaemic control and self-management behaviours in type 2 diabetes: Results from a 1-year longitudinal cohort study. Diabet Med 2015;32(9):1247-54. doi: 10.1111/ dme.12686.

18. Mohebi S, Azadbakht L, Feizi A, Sharifirad G, Kargar M. Review the key role of self-efficacy in diabetes care. J Educ Health Promot 2013;2:36. doi: 10.4103/2277-9531.115827.

19. Senecal C, Nouwen A, White D. Motivation and dietary self-care in adults with diabetes: Are self-efficacy and autonomous self-regulation complementary or competing constructs? Health Psychol 2000;9(5):452-57. doi: 10.10371/02786133.19.5.452.

20. L'Heveder R, Nolan T. International Diabetes Federation. Diabetes Research and Clinical Practice 2013;101(3):349-51. doi: 10.1016/j. diabres.2013.08.003

21. Charmaz K. Constructing grounded theory: A practical guide through qualitative analysis. London: Sage Publications, 2006.

22. Attride-Stirling J. Thematic network: An analytic tool for qualitative research. Qual Res 2001;1(3):385-405. doi: 10.1177/146879410100100307.

23. Raaijmakers LGM, Hamers FJM, Martens MK, Bagchus C, de Vries NK, Kremers SPJ. Perceived facilitators and barriers in diabetes care: $A$ qualitative study among healthcare professionals in the Netherlands. BMC Fam Pract 2013;14:114. doi: 10.1186/1471-2296-14-114.

24. Alhyas L, Nielsen JD, Dawoud D, Majeed A. Factors affecting the motivation of healthcare professionals providing care to Emiratis with type 2 diabetes. JRSM Short Rep 2013;4(2):14. doi: 10.1177/2042533313476419.

25. Brundisini F, Vanstone M, Hulan D, DeJean D, Giacomini M. Type 2 diabetes patients' and providers' differing perspective on medication noadherence: A qualitative meta-synthesis. BMC Health Serv Res 2015;15:516. doi: 10.1186/s12913015-1174-8.

26. Blonde L, Aschner P, Bailey C, et al. Gaps and barriers in the control of blood glucose in people with type 2 diabetes. Diab Vasc Dis Res 2017;14(3):172-83. doi: 10.1177/1479164116679775.

27. Wens J, Vermeiere E, Royen, PV, Sabbe, B, Denekens J. GPs' perspectives of type 2 diabetes patients' adherence to treatment: A qualitative analysis of barriers and solutions. BMC Fam Pract 2005;6(1):20. doi: 10.1186/1471-2296-6-20.

28. Shigaki C, Kruse RL, Mehr D, et al. Motivation and diabetes self-management. Chronic IIIn 2010;6(3):202-14. doi: 10.1177/1742395310375630.

29. Holt RI, Nicolucci A, Kovacs Burns K, et al. Diabetes Attitudes, Wishes and Needs second study $\left(D^{2} \mathrm{WW}^{\mathrm{TM}}\right.$ ): Cross-national comparisons on barriers and resources for optimal care Healthcare professional perspective. Diabet Med 2013;30(7):789-98. doi: 10.1111/dme.12242.

30. Von Korff M, Gruman J, Schaefer J, Curry SJ, Wagner EH. Collaborative management of chronic illness. Ann Intern Med 1997;127(12):1097-102. doi: 10.7326/0003-4819-127-12-199712150-00008. 
31. Anderson RM, Funnel MM, Butler PM, Arnold MS, Fitzgerald JT, Feste CC. Patient empowerment: Results of a randomized controlled trial. Diabetes Care 1995;18(7):943-49. doi: 10.2337/ diacare.18.7.943.

32. Chen K. Fiji national workshop on traditional medicine: mission report 25-31 March 2001. Manila, WHO Regional Office for the Western Pacific, 2001.

33. Modak M, Dixit P, Londhe J, Ghaskadbi S Devasagayam TPA. Indian herbs and herbal drugs used for the treatment of diabetes. J Clin Biochem Nutr 2007;40(3):163-73. doi: 10.3164/jcbn.40.163.

34. Smalls BL, Walker RJ, Bonilha HS, Campbell JA, Egede LE. Community interventions to improve glycaemic control in African Americans with type 2 diabetes: A systemic review. Glob J Health Sci 2015;7(5):171-82. doi: 10.5539/gjhs.v7n5p171.

35. Peimani M, Monjazebi F, Ghodssi-Ghassemabadi R, Nasli-Esfahani E. A peer support intervention in improving glycemic control in patients with type 2 diabetes. Patient Educ Couns 2018;101(3)460-66. doi: 10.1016/j.pec.2017.10.007.

36. Walker J, Chaar BB, Vera N, et al. Medicine shortages in Fiji: A qualitative exploration of stakeholders' views. PloS One 2017:12(6):e0178429. doi: 10.1371/journal. pone. 0178429

37. World Health Organization and Ministry of Health. Health service delivery profile, Fiji, 2012. Manila: WHO, 2012. 